\title{
Studying the Information Content of TMDs using Monte Carlo Generators
}

\author{
H. Avakian \\ Jefferson Laboratory, 12000 Jefferson Avenue, Newport News, VA 23606, USA \\ E-mail: avakian@jlab.org
}

H. Matevosyan

CoEPP and CSSM, School of Chemistry and Physics, The University of Adelaide, Adelaide SA 5005, Australia.

E-mail: hrayr.matevosyan@adelaide.edu.au

\section{B. Pasquini}

University of Pavia, Department of Physics, and INFN-Pavia, 27100 Pavia, Italy

E-mail: barbara.pasquini@pv.infn.it

\section{P. Schweitzer}

University of Connecticut, Department of Physics, Storrs, CT 06269, USA

E-mail: peter.schweitzer@phys.uconn.edu

\begin{abstract}
Theoretical advances in studies of the nucleon structure have been spurred by recent measurements of spin and/or azimuthal asymmetries worldwide. One of the main challenges still remaining is the extraction of the parton distribution functions, generalized to describe transverse momentum and spatial distributions of partons from these observables with no or minimal model dependence. In this topical review we present the latest developments in the field with emphasis on requirements for MonteCarlo event generators, indispensable for studies of the complex 3D nucleon structure, and discuss examples of possible applications.
\end{abstract}

PACS numbers: 12.39.St; 12.39.-x; 13.60.-r; 13.88.+e; 21.60.Ka; 25.30.Rw 


\section{Introduction}

In the modern Standard Model of particle physics the nucleon is a complex object composed of interacting partons (quarks and gluons). The study of the partonic structure of nucleons is one of the main goals of nuclear physics. In a frame where the nucleon has very large momentum, the momenta of partons are almost collinear with the nucleon momentum (Fig.1, left panel), so that the nucleon can be approximated by a stream of partons, each carrying a fraction $x$ of the nucleon momentum. The number densities of partons carrying a momentum fraction $x$ are described by Parton Distribution Functions (PDFs). At large values of $x$ PDFs are dominated by "valence" quarks, which determine the quantum numbers of hadrons, such as electric charge and isospin. In the last decades Deep Inelastic Scattering (DIS) of leptons off nucleons, emerged as a major tool for studying PDFs. Quantum Chromodynamics (QCD), the theory describing the strong interactions, provides crucial guidance for the phenomenology of PDFs in the shape of factorization theorems and evolution equations describing the dependence of PDFs on the hard scale $Q$, which in DIS is associated with the negative four-momentum transfer to the nucleon. However, QCD is not (yet) able to make definitive predictions of the $x$ dependence of PDFs at a given scale due to their non-perturbative nature, which eventually have to be extracted from the data. Standard global fitting methodologies and error analysis have been developed during the decades of PDF measurements [1-4]. Recent studies have shown that especially in the region of large $x$, where little or no direct experimental information is available, the uncertainty related to the choice of parametrization and methodology may be as large or larger than the statistical uncertainty [5,6]. Studies by HERAPDF [5] show that already for $x>0.2$ the parametrization uncertainty for unpolarized PDFs is bigger than the statistical uncertainty. Similar conclusion was derived from the studies of polarized PDFs using different input parametrizations [6]. The bias due to the choice of the fit Ansatz can in principle be avoided using neural networks [7-9], but that technique requires a lot of data and has not yet been used for systematic studies of the multidimensional structure relevant for the understanding of the spin-orbit correlations. This indicates that understanding the dynamics is of importance already for the studies
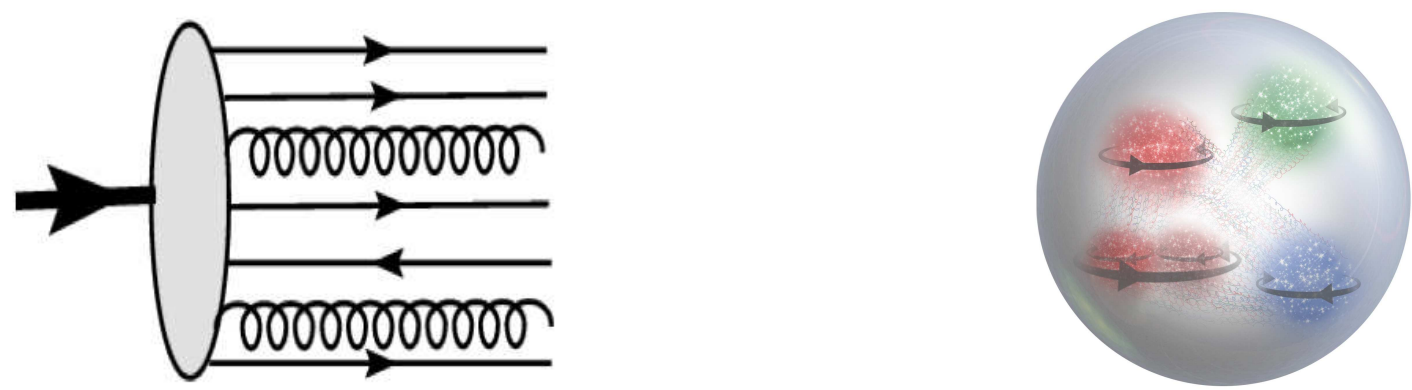

Figure 1. The proton in 1D (left) and 3D (right). 
of 1D PDFs, and more so for the studies of the 3D nucleon structure (Fig.1, right panel). $3 \mathrm{D}$ information on the nucleon is encoded in the transverse momentum dependent parton distributions (TMDs) and the generalized parton distributions (GPDs). As a generalization of PDFs, these functions have initiated a new phase in our exploration of the nucleon, and help to address long-standing questions concerning the orbital motion of partons inside the nucleon, and their spin and spatial distributions. The recent past has witnessed enormous progress in theoretical and experimental methods for the description of these functions. In this review, we summarize the latest developments, and discuss the highest-priority tasks to be addressed in the near future to advance the understanding of the transverse momentum and spatial structure of hadrons. Among these we will discuss the importance of developing Monte Carlo event generators capable of treating transverse and spin degrees of freedom, mainly focusing on the region of relatively large $x$, dominated by valence quarks and non-perturbative sea.

\section{MC Generators}

Monte Carlo event generators are essential components of almost all experimental analyses, and are widely used to make predictions and preparations for future experiments. The models used for underlying event generation and their reliability are important for the understanding of different systematic effects in studies of a variety of observables. The generated final-state particles can be fed into the detector simulation, allowing a precise prediction and verification for the entire experimental setup. However, as the detector simulation is usually a complex and computationally expensive task, simple event analysis techniques are also applied directly to event generator results.

So far simulations of high energy processes were focused primarily on the general features of parton showers, multiparton interactions and hadronization. There are two main classes of event generators, serving different purposes. Main applications include estimating rates of certain types of events, minimizing signal to background conditions, planning and optimizing detector performances, unfolding measured data for acceptance and detector resolution effects, and developing analysis frameworks. Most of the applications typically require full event generators where sets of outgoing particles are produced in the interactions between two incoming particles and a complete event is generated attempting to reproduce the raw data. In certain cases the single particle generators, where only the final state particles of interest are generated, can be used, providing fast tests of analysis procedures with relatively simple integration of different input models.

The most prominent examples of full event generators used by the high energy community include PYTHIA [10,11], HERWIG [12] and SHERPA [13]. Complete event generator programs are based on a combination of analytical results and various QCDbased models, and in general require a detailed modeling of the hadronization process in which colored partons are transformed into jets of colorless hadrons, photons, and leptons. PYTHIA in particular uses the so-called string fragmentation model developed 
as an explicit and detailed framework, where the long-range confinement forces are allowed to distribute the energies and the flavors of a parton configuration among a collection of primary hadrons, which subsequently may decay further. In SHERPA the emission of additional QCD partons off the initial and final states is described through a parton-shower model. The fragmentation of partons into primary hadrons is described using a phenomenological cluster-hadronization model. HERWIG, a generator for simulation of hadronic final states in lepton-lepton, lepton-hadron and hadronhadron collisions, incorporates important color coherence effects in the final state and initial state parton showers. A library of one dimensional PDFs, LHAPDF, has been developed to account for the latest advances in studies of PDFs [14].

The generators developed by the high energy community over the past decades describe well most of the features seen in experiments dedicated to PDF studies. Spin in hard scattering is a very challenging concept to implement in a consistent way. As a result, standard generators fail to describe the new class of measurements, which focuses on azimuthal distributions of final state hadrons and requires detailed understanding of the complex dynamics of spin-orbit correlations.

\section{Generalizing partonic distributions}

Recent measurements of large spin and azimuthal asymmetries worldwide [15-19] cannot be described in a 1D framework and are attributed to orbital motion of quarks, which requires an extension of PDFs to include transverse degrees of freedom. This is done in two complementary ways. GPDs [20-23] describe spatial distributions of partons, which carry the longitudinal momentum fraction $x$, in the transverse ("impact parameter") plane [24]. TMDs [25-28] describe partons with longitudinal momentum fraction $x$ and transverse momentum $k_{T}$. Model examples for each are shown in Fig. 2.

Studies of the distributions of partons in coordinate- and momentum-space as encoded in GPDs and TMDs respectively, have been widely recognized as key objectives of the JLab $12 \mathrm{GeV}$ upgrade and the polarized $p p$ program at RHIC, and are a key
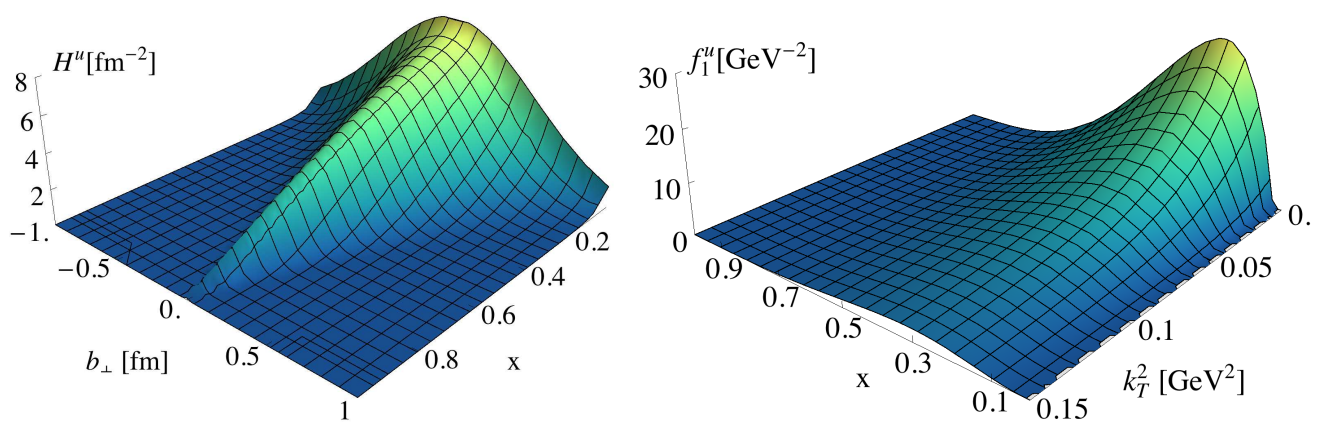

Figure 2. Left panel: distributions of unpolarized up quark as function of $x$ and impact parameter $b_{\perp}$ from Ref. [24]. Right panel: distribution of unpolarized up quark as function of $x$ and the squared quark transverse momentum from Ref. [29]. 
part of the physics program of an Electron Ion Collider (EIC). Data on spin-azimuthal distributions of hadrons in semi-inclusive DIS (SIDIS) and polarized hadron-hadron collisions, which provide access to TMDs, have been accumulated in recent years by several collaborations, including HERMES, COMPASS, BELLE, BaBar, Halls A, B, C at JLab, and PHENIX and STAR at RHIC (BNL). After the first years of JLab12 and the upcoming polarized $p p$ runs at RHIC, significantly larger data sets will be available. Although the interest in TMDs has grown enormously, we are still in need of a consistent theoretical and phenomenological description spanning the full kinematic regime covered by the (un)polarized world-data. Some TMDs have been already phenomenologically extracted, mainly from analyzing azimuthal distributions of single hadrons in SIDIS. To obtain a full picture about the 3D momentum structure of the partons in the nucleon from high to low $x$, it is important to connect the theoretical approaches to extract TMDs including evolution. The studies of 3D PDFs in general, and TMDs in particular, require a lot more attention to uncertainties due to input parametrizations, as more degrees of freedom and bigger number of input parameters may generate uncontrolled model uncertainties. This makes the development of a framework for testing different extraction procedures a high priority task for the community involved in studies of 3D PDFs. Development of realistic Monte-Carlo generators accounting for TMD evolution effects, spin-orbit and quark-gluon correlations will be a great support for a multifaceted effort to study the fundamental 3D structure of matter.

\section{Azimuthal distributions of hadrons in electroproduction}

Calculation of production cross sections rely upon knowledge of the distribution of the momentum fraction $x$ and transverse momentum $k_{T}$ of partons (quarks and gluons) in incoming hadrons in the relevant kinematic range. These parton densities cannot be calculated perturbatively, but rather are determined by global fits to data from SIDIS, Drell-Yan (DY), and, if applicable, from jet production at energy ranges of interest. In addition to having the best estimates for the values of the TMDs in a given kinematic range, it is important to understand their uncertainties. The partonic content of hadrons needs to be well understood and of sufficiently high precision if theoretical predictions are to match the experimental accuracy expected from future high precision measurements.

We consider the SIDIS process $\ell(l)+N(P) \rightarrow \ell\left(l^{\prime}\right)+h\left(P_{h}\right)+X$ where $\ell$ denotes the lepton, $N$ the nucleon, and $h$ the produced hadron with four-momenta given in parentheses. The four-momentum transfer is $q=l-l^{\prime}$ with $Q^{2}=-q^{2}$. The relevant SIDIS variables are defined as $x=Q^{2} /(2 P \cdot q), y=(P \cdot q) /(P \cdot l), z=\left(P \cdot P_{h}\right) /(P \cdot q)$. $P_{h \perp}$ is momentum component of the produced hadron transverse with respect to virtual

photon with $P_{h \perp}=\left|P_{h \perp}\right|$. Assuming single photon exchange, the lepton-hadron cross section is expressed model-independently by a set of structure functions, see e.g. [30],

$$
\frac{d \sigma^{e^{-} N \rightarrow e^{-} h X}}{d x d y d \phi_{s} d z d \phi_{h} d P_{h \perp}^{2}}=\frac{\alpha_{e m}^{2}}{2 x y Q^{2}} \frac{y^{2}}{1-\varepsilon}\left(1+\frac{\gamma^{2}}{2 x}\right)
$$




$$
\begin{aligned}
& \times\left\{F_{U U, T}+\varepsilon F_{U U, L}+\sqrt{2 \varepsilon(1+\varepsilon)} \cos \phi_{h} F_{U U}^{\cos \phi_{h}}+\varepsilon \cos \left(2 \phi_{h}\right) F_{U U}^{\cos 2 \phi_{h}}\right. \\
& +\lambda_{e} \sqrt{2 \varepsilon(1-\varepsilon)} \sin \phi_{h} F_{L U}^{\sin \phi_{h}} \\
& +S_{L}\left[\sqrt{2 \varepsilon(1+\varepsilon)} \sin \phi_{h} F_{U L}^{\sin \phi_{h}}+\varepsilon \sin \left(2 \phi_{h}\right) F_{U L}^{\sin 2 \phi_{h}}\right] \\
& +\lambda_{e} S_{L}\left[\sqrt{1-\varepsilon^{2}} F_{L L}+\sqrt{2 \varepsilon(1-\varepsilon)} \cos \phi_{h} F_{L L}^{\cos \phi_{h}}\right] \\
& +S_{T}\left[\sin \left(\phi_{h}-\phi_{S}\right)\left(F_{U T, T}^{\sin \left(\phi_{h}-\phi_{S}\right)}+\varepsilon F_{U T, L}^{\sin \left(\phi_{h}-\phi_{S}\right)}\right)\right. \\
& \quad+\varepsilon \sin \left(\phi_{h}+\phi_{S}\right) F_{U T}^{\sin \left(\phi_{h}+\phi_{S}\right)}+\varepsilon \sin \left(3 \phi_{h}-\phi_{S}\right) F_{U T}^{\sin \left(3 \phi_{h}-\phi_{S}\right)} \\
& \left.\quad+\sqrt{2 \varepsilon(1+\varepsilon)} \sin \phi_{S} F_{U T}^{\sin \phi_{S}}+\sqrt{2 \varepsilon(1+\varepsilon)} \sin \left(2 \phi_{h}-\phi_{S}\right) F_{U T}^{\sin \left(2 \phi_{h}-\phi_{S}\right)}\right] \\
& +\lambda_{e} S_{T}\left[\sqrt{1-\varepsilon^{2}} \cos \left(\phi_{h}-\phi_{S}\right) F_{L T}^{\cos \left(\phi_{h}-\phi_{S}\right)}+\sqrt{2 \varepsilon(1-\varepsilon)} \cos \phi_{S} F_{L T}^{\cos \phi_{S}}\right. \\
& \left.\left.\quad+\sqrt{2 \varepsilon(1-\varepsilon)} \cos \left(2 \phi_{h}-\phi_{S}\right) F_{L T}^{\cos \left(2 \phi_{h}-\phi_{S}\right)}\right]\right\}
\end{aligned}
$$

where $\alpha_{e m}$ is the fine structure constant, and $\phi_{h}\left(\phi_{S}\right)$ is the azimuthal angle of the produced hadron (azimuthal angle of transverse target polarization). On the structure functions $F_{X Y, Z}^{\text {weight }}=F_{X Y, Z}^{\text {weight }}\left(x, Q^{2}, z, P_{h \perp}^{2}\right)$, the subscripts $X, Y=\{U, L, T\}$ indicate the respective polarizations of beam and target, and $Z=\{L, T\}$ indicates polarization of the virtual photon where needed. The superscript "weight" reminds us of the kind of angular distribution of the produced hadrons. Furthermore, the ratio of longitudinal to transverse photon flux is $\varepsilon=\left(1-y-\frac{1}{4} \gamma^{2} y^{2}\right) /\left(1-y+\frac{1}{2} y^{2}+\frac{1}{4} \gamma^{2} y^{2}\right)$ with $\gamma=2 M x / Q$. The polarization of the lepton is described by the helicity $\lambda_{e}$, and $\left(\boldsymbol{S}_{T}, S_{L}\right)$ is the nucleon polarization vector with $S_{T}=\left|\boldsymbol{S}_{T}\right|$.

Depending on the kinematics, the structure functions can be interpreted in terms of transverse-momentum dependent parton distribution and fragmentation functions, or in terms of the equally interesting but so far less studied fracture functions. By exploring different beam and target polarizations, and detecting a variety of final state hadrons (pions, kaons) one can access a wealth of novel information about the 3D spin structure of the nucleon, as well as spin-orbit effects in the target fragmentation region [30,31].

Following the TMD factorization theorem one can write SIDIS structure functions in the current fragmentation region as a convolution of perturbatively calculable part $\mathcal{H}^{a}\left(Q^{2}\right)$, and universal TMD PDFs and TMD fragmentation functions [32-35]

$$
\begin{aligned}
& F_{U U, T}\left(x, z, P_{h \perp}^{2}, Q^{2}\right)=\sum_{a} \mathcal{H}^{a}\left(Q^{2}\right) \iint d \boldsymbol{k}_{T} d \boldsymbol{p}_{T} f_{1}^{a}\left(x, k_{T}^{2} ; Q^{2}\right) \\
& \quad \times D_{1}^{a \rightarrow h}\left(z, p_{T}^{2} ; Q^{2}\right) \delta\left(z \boldsymbol{k}_{T}+\boldsymbol{p}_{T}-\boldsymbol{P}_{h \perp}\right)+Y\left(Q^{2}, P_{h \perp}^{2}\right)+\mathcal{O}(M / Q) \\
& =\sum_{a} \mathcal{H}^{a}\left(Q^{2}\right) \int d^{2} \boldsymbol{b}_{T} e^{i \boldsymbol{b}_{T} \cdot \boldsymbol{P}_{h \perp}} \tilde{f}_{1}^{a}\left(x, b_{T} ; Q\right) \tilde{D}_{1}^{a \rightarrow h}\left(z, b_{T} ; Q\right)+\ldots
\end{aligned}
$$

and analog for polarized structure functions [36]. Here $f_{1}^{a}\left(x, k_{T}^{2} ; Q^{2}\right)$ is the unpolarized TMD PDF describing an unpolarized parton of flavor $a$ carrying longitudinal momentum fraction $x$ and transverse momentum $k_{T}$ at the renormalization scale chosen to be equal to $Q^{2}$. $D_{1}^{a \rightarrow h}\left(z, p_{T}^{2} ; Q^{2}\right)$ is the TMD fragmentation function for an unpolarized parton of flavor $a$ fragmenting into an unpolarized hadron $h$ which carries the longitudinal momentum fraction $z$ and acquires the transverse momentum $p_{T}$ (with respect to the fragmenting quark) during the fragmentation process. The term $Y$ is introduced to 
ensure a matching to the perturbative calculations at high transverse momentum. In the last step of Eq. (2) a 2D Fourier transform to express the TMDs in terms of the transverse coordinate $b_{T}$ was used, which facilitates the discussion of evolution. At short distances $b_{T} \ll 1 / \Lambda_{\mathrm{QCD}}$ where $\alpha_{s}\left(1 / b_{T}\right)$ is small, one can use perturbative QCD. But at large $b_{T}$ one is sensitive to details of the hadron wave function and a perturbative treatment is not possible. The practical prescription to tame the growth of $\alpha_{s}\left(1 / b_{T}\right)$ and match to a non-perturbative large distance description of the $b_{T}$-dependence consists in freezing the renormalization scale $\mu_{b}$ as

$$
\mu_{b}=C_{1} / b_{*}\left(b_{T}\right) \quad \text { with } \quad b_{*}\left(b_{T}\right)= \begin{cases}b_{T} & \text { for } b_{T} \ll b_{\max }, \\ b_{\max } & \text { for } b_{T} \gg b_{\max },\end{cases}
$$

which ensures a sufficiently large $\mu_{b}$ and applicability of perturbative QCD. The choice $C_{1}=2 e^{-\gamma_{E}}$ for the arbitrary constant $C_{1}$ helps to minimize higher order corrections. One popular taming prescription (though other choices are also possible) is

$$
b_{*}\left(b_{T}\right)=b_{T} / \sqrt{1+b_{T}^{2} / b_{\max }^{2}} .
$$

Identifying renormalization and factorization scales and the scales regulating lightcone divergences with the hard scale $Q$ of the process, the TMD evolved from $\mu_{b}$ to $Q$ is

$$
\tilde{f}_{1}\left(x, b_{T} ; Q\right)=\tilde{f}_{1}\left(x, b_{T} ; \mu_{b}\right) e^{-g_{f}\left(x, b_{T} ; b_{\max }\right)-g_{K}\left(b_{T} ; b_{\max }\right) \ln \frac{Q}{\mu_{b}}} \Phi,
$$

where $\Phi=\Phi\left(b_{*}\left(b_{T}\right), \mu_{b}, Q\right)$ is perturbatively calculable. The function $g_{f}$ is specific for a given TMD (or fragmentation function), while $g_{K}$ is flavor-independent and describes universal soft-gluon radiation. The arbitrariness in choosing the taming prescription and the numerical value $b_{\max }$ is compensated by redefining correspondingly the nonperturbative functions $g_{f}\left(b_{T} ; b_{\max }\right)$ and $g_{K}\left(x, b_{T} ; b_{\max }\right)$. In practical applications it is nevertheless important to choose a physically well-motivated $b_{\max }$ in order to ideally exploit the physics output from a TMD analysis [37-44]. The scale $\mu_{b}$ could in principle be chosen as the initial scale of a model for TMDs. The MC generator accounting for spin-orbit correlations, which has been discussed recently on a dedicated workshop at INT Seattle [45], should account for latest developments in TMD theory and include all known scales, parameters and functions listed above as user defined input.

\section{Nonperturbative properties of TMDs}

The current knowledge of TMDs at relatively large $\mathrm{x}$, where the spin-orbit correlations are significant, is based on theoretical studies in quark models, first lattices studies, and phenomenological extractions, which are reviewed below.

\subsection{Information From First Principles}

TMDs are subject to positivity constraints [46]. In the limit of a large number of colors $N_{c}$ the $(u \pm d)$ and $(\bar{u} \pm \bar{d})$ flavor combinations of TMDs exhibit different behavior [47]. Such large- $N_{c}$ scaling relations are well-supported by phenomenology [48] (see 
below). First attempts to access information on Mellin moments of TMDs in Euclidean space-time lattice QCD simulations were reported [49-51]. One of the most exciting predictions from lattice studies were observations of strong spin and flavor dependences of $k_{T}$-distributions of partons [50,51]. Recently a new approach was proposed to access also the $x$-dependence of PDFs and TMDs [52,53] from lattice QCD. More work is needed [54], but the first calculations [55] indicate feasibility of the method.

\subsection{TMD Models}

TMDs were studied in various low-energy QCD-inspired models often based on complementary assumptions. Models oversimplify the complexity of QCD dynamics. Still, results from diverse models provide valuable insights to non-perturbative aspects. From the point of view of modeling, it is important to distinguish between T-even and T-odd TMDs according to their behavior under "naive time reversal", which is defined as usual time reversal but without interchange of initial and final states. The modeling of T-odd TMDs requires explicit gauge-field degrees of freedom, while for T-even TMDs it is sufficient to consider quark degrees of freedom.

Models of T-even TMDs can be classified as follows: (a) spectator models [56-61]; (b) light-front models [29,62-64]; (c) chiral quark soliton models [65-67]; (d) bag models [68,69]; (e) covariant parton models [70-72]; (f) quark-target models [73-77].

(a) In quark-diquark spectator models [56], one evaluates the correlation function that defines TMDs by truncating the sum over all intermediate states to a single on-shell spectator with quantum numbers of a scalar isoscalar or axial-vector isovector diquark. The diquark is understood as an effective particle which resumes nonperturbative effects related to sea quarks and gluons, and allows to treat the complicated many-particle system effectively by simple two-particle technique. A difficulty is that momentum and quark-number sum rules cannot be satisfied simultaneously, unless one considers the diquark as composed of two quarks [78]. Another approach free of these difficulties consists of describing scalar and axial-vector diquark correlations by a relativistic Faddeev equation in the Nambu-Jona-Lasinio model [79]. This model predicts transverse-momentum dependencies that are softer than a Gaussian fall-off, which was also recently seen in preliminary COMPASS data [80].

(b) Light-front models are built by expanding the nucleon state in the basis of free parton (Fock) states. One typically truncates the expansion to the minimal Fock state, which can correspond to three valence quarks in the light-front constituent quark model [29] or a valence quark and spectator diquark in the light-front quark-diquark model [62-64]. The probability amplitude to find these configurations in the nucleon is given by light-front wave functions which encode the bound state properties of hadrons, and have the important property to be eigenstates of the total quark orbital-angular momentum $L_{z}^{q}[81,82]$. This allows one to map the multipole patterns in $k_{T}$ associated with each TMD $[29,83,84]$. For phenomenological applications the $k_{T}$ dependence in these approaches can be usefully approximated by a Gaussian form [63, 84-87]. 
(c) The chiral quark soliton model was applied to describe unpolarized $[65,66]$ and helicity [66] TMDs of quarks and antiquarks. It is the only model so far including sea quarks, and predicts that sea-quark TMDs have significantly broader $k_{T}$-distributions than valence quarks, due to nonperturbative short-distance correlations caused by chiral symmetry breaking effects [66]. All T-even TMDs were predicted within the light-front version of this model restricted to the 3-quark light-front Fock state [67].

(d) The bag model is the only model discussed so far which incorporates confinement. It has been applied to calculate all twist-2 and twist-3 T-even TMDs in $[68,69]$, and a complete set of linear and non-linear relations among them was derived (see also Ref. [88] for a discussion of the validity of such relations in quark models). Another remarkable insight is that the bag model strongly supports the Gaussian $k_{T^{-}}$ dependence of TMDs observed in phenomenology [89].

(e) In the covariant parton model [70-72] the partons are free, and described in terms of two covariant 3D momentum distributions which are spherically symmetric in nucleon rest frame: one describes unpolarized and the other polarized quarks. This model predicts the $x$ - and $k_{T}$-dependence of TMDs from the $x$-dependence of PDFs [72] at large scales. Also this model supports an approximate Gaussian $k_{T^{-}}$dependence. Other parton model approaches in the context of TMDs were discussed in [90-92].

(f) In quark-target models [73-77] the nucleon target is replaced by quark target. These are strictly speaking not nucleon models. But their QCD-type quark and gaugefield degrees of freedom make these models valuable theoretical test grounds, e.g. in [73] the limitation of the Lorentz-invariance relations was recognized (cf. [93] for a review).

In order to model T-odd TMDs gauge-boson degrees of freedom are required to describe initial/final-state interactions (ISI/FSI) between struck parton and target remnants. At leading twist there are two T-odd nucleon TMDs, the Sivers function and Boer-Mulders function. The first calculation which explicitly predicted a non-zero Sivers function within a scalar-diquark model [94] was followed by studies of T-odd TMDs in spectator models [57,58,60,74,95-98], bag models [99-101], non-relativistic model [102] and a light-front constituent quark model [103] (see Fig. 3 for a result from the latter). In these models the FSI/ISI are approximated by one-gluon exchange mechanism. The final expressions for T-odd functions are proportional to the strong coupling constant, which depends on the intrinsic hadronic scale of the model. Non-perturbative eikonal methods to calculate higher-order gluonic contributions from the gauge link were applied in spectator frameworks to improve this approximation $[59,104,105]$. A complementary approach is to construct augmented light-front wave functions which incorporate the rescattering effects by acquiring an imaginary (process-dependent) phase [106].

Although nucleon structure is the main topic, we include a brief review of TMD fragmentation functions which are of interest by themselves, besides their role for the interpretation of SIDIS data. Model studies of the T-odd Collins function were pioneered in [107] for pions using the Lund string mechanism, which was applied to vector mesons [108], and extended to include quark spin [109]. T-even pion and proton fragmentation functions were studied in [56] within a spectator model. The 
Collins fragmentation function was studied in spectator-type frameworks with final state interactions modeled by rescattering among the quark fragments [110-114] or one-gluon exchange [115]. Twist-2 pion and kaon fragmentation functions were investigated in the Nambu-Jona-Lasinio-jet model using Monte Carlo simulations [79, 116], including favored and unfavored Collins functions [117,118]. A review of fragmentation functions (including the collinear interference fragmentation functions not discussed here) can be found in [119].

\subsection{First extractions of TMDs}

Compared to PDFs, the status of TMD extractions is still in an "exploratory stage." Admittedly, in the Collins-Soper-Sterman (CSS) framework [122] parametrizations of the $k_{T}$-dependence of unpolarized TMDs are routinely extracted from collider data on electroweak boson production [123-127] with important applications for Higgs production at LHC and jet physics, e.g. [128-130]. In these fits the entire richness of the 3D nucleon structure is "dumped" into 2 parameters assuming a fit form $g_{f}\left(x, b_{T}\right)=\left(g_{1}+\ln \frac{Q}{2 \mu_{b}}\right) b_{T}^{2}$ [123] (or 3 parameters [124,125] using similar fit forms). This corresponds (roughly) to a Gaussian Ansatz, leaves no room for flavor dependence, and satisfactorily describes $W$ - and $Z$-boson spectra, showing that these collider data have limited (though undeniable [131,132]) sensitivity to the details of 3D nucleon structure.

There are open questions even in this rather well-established field. E.g. when using the $b_{*}\left(b_{T}\right)$ prescription (3) excellent fits to global DY and $Z$ boson production data were obtained with very different choices of $b_{\max }=0.1 \mathrm{fm}[126]$ and $b_{\max }=0.3 \mathrm{fm}$ [127]. The first value is perhaps too small and in perturbative regime. The second value seems physically more appropriate to mark the transition between perturbative and non-perturbative regimes as argued in [40] and supported by chiral physics [66].

First efforts to extract information on novel TMDs concentrated on Sivers function [120, 121, 133-136], transversity [136-139] or Boer-Mulders function [140-144], and included tests of theoretical large- $N_{c}$ predictions $[121,133]$. Fig. 3 shows extractions

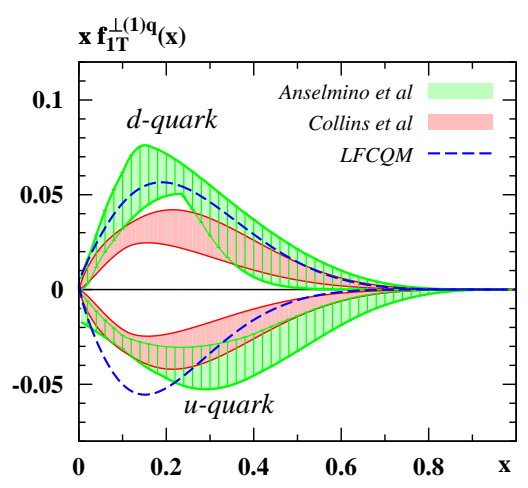

Figure 3. Extractions of (1)-transverse moments of the Sivers functions $x f_{1}^{\perp q}(x)$ by Anselmino et al [120] and Collins et al [121] at a scale of $2.5 \mathrm{GeV}^{2}$. The theoretical calculation is from the light-front constituent quark model (LFCQM) $[85,103]$. 
of the Sivers function by different groups (cf. [145]) from basically the same SIDIS data from HERMES. During the past decade the error bars have decreased, but discrepancies due to assumed fit functions and employed methodologies remain. In these studies TMD evolution was neglected or crudely estimated, with uncontrolled impact on DY [146-151]. With the advent of TMD evolution equations, advances towards more precise quantitative treatments became possible [37, 38,41-43, 152-158]. Further progress will also be driven by data, such as the recent COMPASS data [159] analyzed in $[160,161]$. Phenomenology efforts have been summarized recently by introduction of a library of fits and parameterisations for transverse-momentum-dependent parton distribution functions (TMD PDFs) and fragmentation functions (TMD FFs) together with an online plotting tool, TMDplotter [162].

\section{MC in theory and practice}

Further progress in the interpretation of the quantitative description of azimuthal, spinorbit, and polarization phenomena in SIDIS at JLab 12 and EIC, and other processes (DY at RHIC, DY at COMPASS) will require novel MC technologies.

\subsection{TMD-MC applications}

Both full event generators and single-particle generators were extensively used for studies of different hard scattering processes and development of analysis frameworks. Several single-hadron and di-hadron generators were extensively used at HERMES, JLab and COMPASS.

Studies of the effects of limited energy on the available phase space to generate high $P_{T}$ SIDIS events have been performed for JLab and COMPASS kinematics using a multidimensional generator [163] starting from the initial transverse momenta of quarks and accounting for the energy conservation across the production process. Fig. 4 (right panel) shows the final distribution, compared to the respective initial input Gaussian distribution. A possible effect of limited phase space may have been also observed by the Torino group [161] in attempts to describe $P_{T}$-distributions measured in JLab Hall-C experiment [164] by using parameters extracted from fits to HERMES multiplicities, as shown in left panel of Fig. 4.

One of the most commonly used MC applications are detector acceptance studies, which may have strong dependences on input distribution and fragmentation functions. Normally, experimental setups have a minimum polar angle for detection, which introduces some azimuthal modulation depending on the kinematical variables. An example of CLAS12 acceptance, defined as the ratio of reconstructed and generated events, for hadrons production in the range of $P_{T}$ from 0.4 to $0.6 \mathrm{GeV}$ is shown in Fig. 5 .

In experiments, one measures a convolution of cross section and detector acceptance. One can describe the detector acceptance $A\left(x, Q^{2}, P_{T}, z, \phi\right)$ with Fourier 

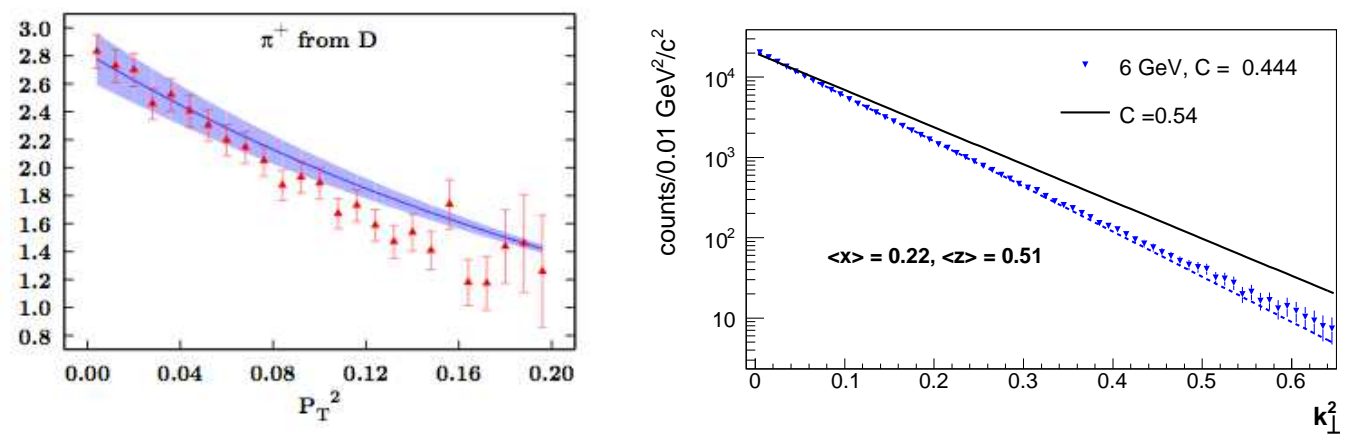

Figure 4. Left panel: JLab Hall-C data [164] on the cross section $d^{5} \sigma / d \Omega d E d z d P_{T}^{2}$ (in units of $\mathrm{nb} / \mathrm{GeV}^{3} \mathrm{sr}$ ) for $\pi^{+}$production from deuterium in comparison to calculations of Ref. [161] based on simple Gaussian approximation, cf. [89]. Right panel: MC generated distributions implementing Gaussian function (solid line). The (blue) down triangles show the outcome for distributions from the $\mathrm{MC}$ at $6 \mathrm{GeV}$ initial lepton energy, in comparison with the fit (dashed curve) with Gaussian function with a smaller width $[163,165]$.

series, where the constants $A_{m}$ and $B_{n}$ depend on the kinematical variables:

$$
A\left(x, Q^{2}, P_{T}, z, \phi\right)=A_{0}\left(1+\sum_{m=1}^{\infty} A_{m} \cos m \phi+\sum_{n=1}^{\infty} B_{n} \sin n \phi\right) .
$$

With knowledge of the acceptance function provided by a realistic MC simulation, one can extract azimuthal moments from the complicated product using the following formulas

$$
\begin{aligned}
& \sigma_{M N}=A(\phi) \sigma(\phi)=A_{0} \sigma_{0}\left\{1+\frac{1}{2}\left(\sum_{i=1}^{M} A_{i} a_{i}+\sum_{i=1}^{N} B_{i} b_{i}\right)\right. \\
& +\cos \phi\left[a_{1}+A_{1}+\frac{1}{2}\left(\sum_{i=1}^{M-1} A_{i} a_{i+1}+\sum_{i=1}^{M-1} A_{i+1} a_{i}+\sum_{i=1}^{N-1} B_{i} b_{i+1}+\sum_{i=1}^{N} B_{i+1} b_{i}\right)\right] \\
& +\sin \phi\left[b_{1}+B_{1}+\frac{1}{2}\left(\sum_{i=1}^{M-1} B_{i+1} a_{i}-\sum_{i=1}^{M-1} B_{i} a_{i+1}+\sum_{i=1}^{N-1} A_{i} b_{i+1}-\sum_{i=1}^{N} A_{i+1} b_{i}\right)\right] \\
& +\ldots\},
\end{aligned}
$$

where $\sigma_{M N}$ is the measured yield parametrized in terms of respectively $M$ and $N$ cosine and sine modulations, and $\sigma_{0}, a_{i}$ and $b_{i}$ correspond to azimuthal moments in the cross section, defined by the corresponding structure functions in Eq. (1).

The acceptance has weak dependence on the input cross sections, but for precision studies of azimuthal distributions this weak dependence may be crucial. In the CLAS12 example shown in Fig. 5, the two initial $\phi$-distributions, $\phi$-independent and $\phi$-dependent with $\langle\cos \phi\rangle=-0.15$, yield $\cos \phi$ moments in the acceptance equal to $5.4 \%$ and $4.5 \%$, respectively. This variation may be much stronger at higher $z$ and $P_{T}$, indicating the importance of a precise description of spin-azimuthal terms for the extraction of different azimuthal moments in hard scattering processes. Accounting for all the relevant 

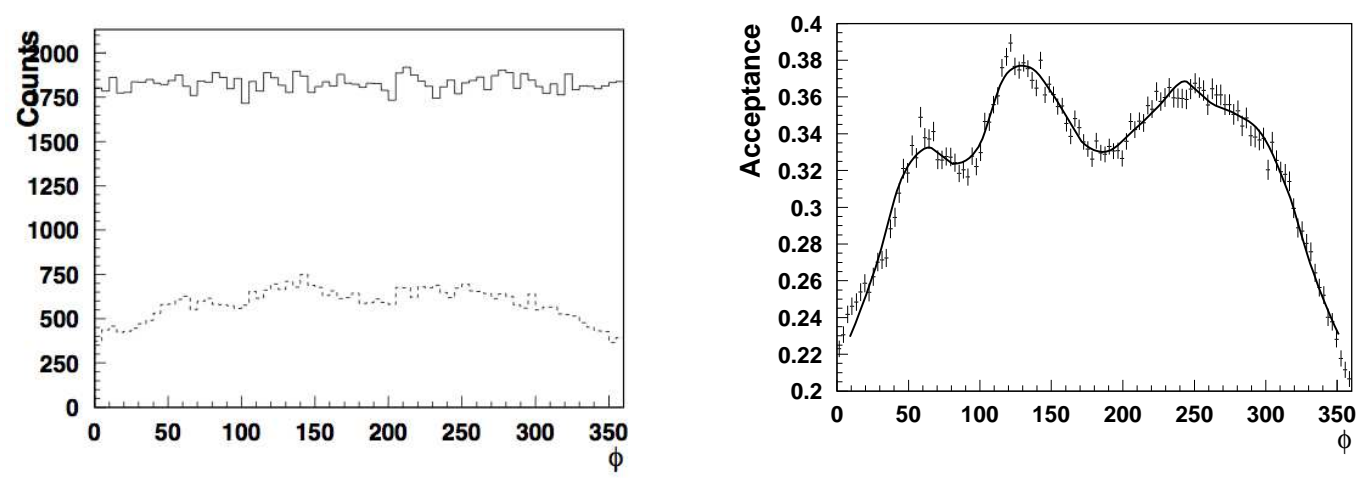

Figure 5. Left panel: generated (flat) and reconstructed $\phi$ distributions in $e p \rightarrow$ $e^{\prime} \pi^{+} X$ events for CLAS12 detector and $11 \mathrm{GeV}$ electron beam energy. Right panel: acceptance (ratio of reconstructed to generated events) fitted with the Fourier series in Eq. (6), including the first ten cosine moments and the first four sine moments.
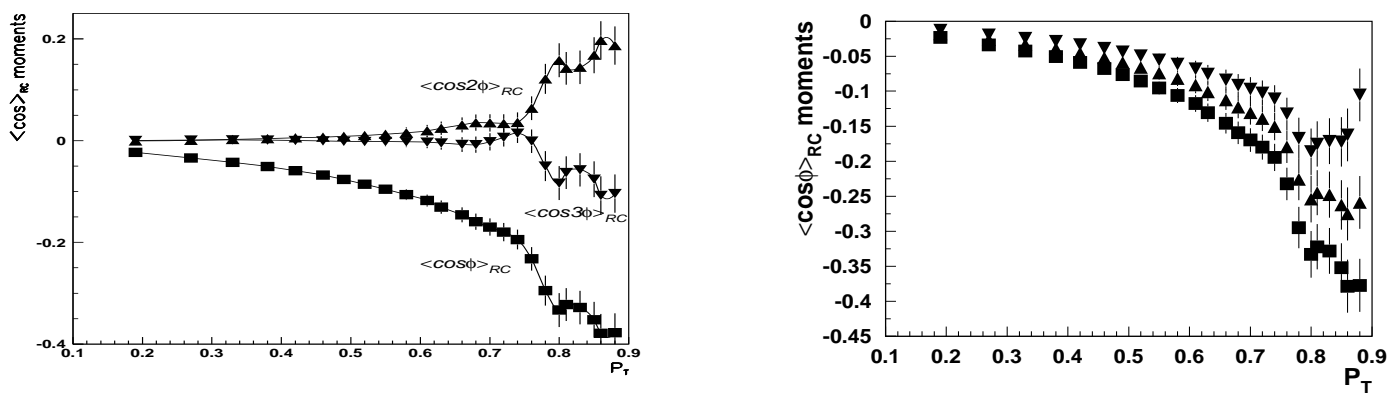

Figure 6. Left panel: Azimuthal modulations in the final cross section generated by radiation of additional photon calculated from haprad2.0 [167] as a function of the transverse momentum of a pion in SIDIS in JLab12 kinematics at $x=0.3$ and $z=0.3$. Right panel: Sensitivity of the $\cos \phi$-moment generated by radiative effects to $\phi$-dependence of three different structure functions with $\cos \phi$ input amplitudes equal to $-5 \%$ (squares), $-10 \%$ (triangles up), and $-15 \%$ (triangles down).

azimuthal moments in the MC generator, it will be crucial also to properly handle the radiative effects. As a matter of fact, the radiative effects in SIDIS also generate azimuthal moments which may couple with the moments in the Born cross section and produce more complex azimuthal structure in the observed cross section $[166,167]$. Azimuthal moments generated by radiative effects, and their sensitivity to different input $\phi$-dependent structure functions are shown on Fig.6.

A good agreement with some existing data on spin-azimuthal asymmetries in the current fragmentation region of SIDIS has been achieved using different TMD MCs. Implementation of spin-orbit correlations in a consistent way will, however, require development of a procedure for proper accounting of spin effects in the distribution and fragmentation processes. 


\subsection{Towards the complete TMD MC}

Complete MC event generators, like LEPTO [168] and PYHTIA [10,11], are invaluable tools for various experimental and phenomenological studies, as mentioned in Sec. 2. Spin effects due to nucleon polarization or polarized partons in the nucleon and fragmentation process are not accounted for. Effects involving unpolarized partons, like Cahn and Sivers azimuthal modulations of the transverse momentum of the active quark before hard scattering and hadronization, can be incorporated in existing codes with small modifications, as done in mLEPTO [169,170] and LEPTO-PHI [171]. Moreover, mLEPTO has been recently used to study the Sivers single spin asymmetries in both single hadron and dihadron SIDIS production in COMPASS kinematics [172,173].

The incorporation of spin effects in the hadronization of polarized partons require much more efforts, and microscopic models of the fragmentation mechanism. Fragmentation of transversely polarized quarks was studied within the string fragmentation framework [174-176]. A stand-alone MC generator based on the NJLjet model was developed in $[117,118]$. The development of a full MC event generator would require consistent models of the hadronization of polarized partons, including the polarization transfer in each hadron emission for cascade-type mechanisms, and the production and strong decays of vector mesons which is of particular importance for dihadron studies [177-179].

A new MC generator could be built extending existing MC generators, e.g., on the basis of the ThePEG framework [180] underlying the HERWIG++ generator. A desirable feature would be the separation of a user-friendly and easily modifiable physics layer from the underlying complex computational layer. The advantage of such a separation would be that developers could maintain and update the underlying algorithms and event generation software, without interfering with the customizations of the users. A realistic MC with solid fundamentals will be important part of the global 3D analysis framework, including the data analysis, phenomenology and extended 3D PDF library (see Fig.7). These and other features of the required MC event generators were vividly discussed at the dedicated INT workshop in Seattle [45].

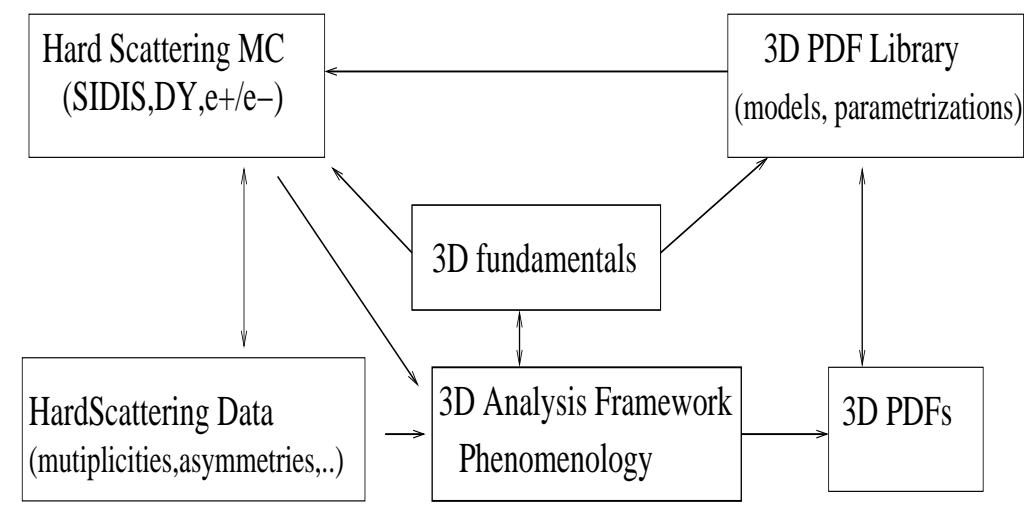

Figure 7. Global analysis framework. 


\section{Conclusions}

The 3D partonic structure accessible in hard scatterings is rich and complex. The understanding of the contributions to the final transverse-momentum dependence of different azimuthal moments in the cross section [181] will require detailed studies. Monte-Carlo event generators accounting for spin-orbit correlations will be crucial to study the dependence on different model inputs, as well as sensitivity of the extraction of underlying TMDs on various experimental uncertainties including acceptances, resolutions and radiative corrections. The future 3D nucleon structure extraction framework, should include in addition to extraction procedures a library of 3D PDFsł and MC simulation frameworks using that library as input and allowing to check the quality of extracted PDFs for specific experimental conditions in a full range of accessible kinematics. This brief review was focused on MC applications to TMD studies, and those concepts that are of direct relevance such as model studies or extractions which provide input for MC generators. Our focus was primarily on spin-orbits effects expected to play a role in the region of not too small $x$. At small $x$ unpolarized and polarized gluon TMDs become relevant, about which little is known and which can be studied in hadron colliders such as RHIC and LHC [182-193]. For these and other fascinating topics that could not be covered due to space limitations we refer to the excellent reviews [194-196].

\section{Acknowledgments}

We thank E. Aschenauer for helpful comments and corrections, and I. Akushevich for clarifying certain aspects of radiative corrections. The authors are indebted to the participants of the INT workshop INT-14-55W "Studies of 3D Structure of Nucleon" [45] for stimulating discussions which inspired parts of this brief review. This work was partially supported by DOE contract No. DE-AC05-06OR23177, under which Jefferson Science Associates, LLC operates Jefferson Lab; the European Community Joint Research Activity "Study of Strongly Interacting Matter" (acronym HadronPhysics3, Grant Agreement No. 283286) under the Seventh Framework Programme of the European Community; the Australian Research Council through Grants FL0992247 (AWT) and No. CE110001004 (CoEPP), and the U.S. National Science Foundation under Contract No. 1406298.

[1] Martin A D, Roberts R, Stirling W and Thorne R 2002 Eur.Phys.J. C23 73-87 (Preprint hep-ph/0110215)

[2] Pumplin J, Stump D, Huston J, Lai H, Nadolsky P M et al. 2002 JHEP 0207012 (Preprint hep-ph/0201195)

[3] Martin A D, Stirling W J, Thorne R S and Watt G 2009 Eur. Phys. J. C63 189-285 (Preprint $0901.0002)$

[4] Lai H L, Guzzi M, Huston J, Li Z, Nadolsky P M et al. 2010 Phys.Rev. D82 074024 (Preprint 1007.2241)

[5] Aaron F et al. (H1 and ZEUS Collaboration) 2010 JHEP 1001109 (Preprint 0911.0884)

$\ddagger$ An important first step towards a library of 3D PDFs is the project "TMDlib and TMDplotter version 1.0.0" which recently became available [162] after this article was completed. 
[6] Jimenez-Delgado P, Avakian H and Melnitchouk W (Jefferson Lab Angular Momentum (JAM) Collaboration) 2014 (Preprint 1403.3355)

[7] Ball R D, Del Debbio L, Forte S, Guffanti A, Latorre J I et al. 2010 Nucl.Phys. B838 136-206 (Preprint 1002.4407)

[8] Ball R D et al. (NNPDF Collaboration) 2012 Nucl.Phys. B855 153-221 (Preprint 1107.2652)

[9] Nocera E R, Ball R D, Forte S, Ridolfi G and Rojo J (The NNPDF Collaboration) 2014 (Preprint 1406.5539)

[10] Sjostrand T, Mrenna S and Skands P Z 2006 JHEP 0605026 (Preprint hep-ph/0603175)

[11] Sjostrand T, Mrenna S and Skands P Z 2008 Comput.Phys.Commun. 178 852-867 (Preprint 0710.3820)

[12] Corcella G, Knowles I, Marchesini G, Moretti S, Odagiri K et al. 2001 JHEP 0101010 (Preprint hep-ph/0011363)

[13] Gleisberg T, Hoeche S, Krauss F, Schonherr M, Schumann S et al. 2009 JHEP 0902007 (Preprint $0811.4622)$

[14] Butterworth J, Dissertori G, Dittmaier S, de Florian D, Glover N et al. 2014 (Preprint 1405.1067)

[15] Bravar A et al. (Fermilab E704) 1996 Phys. Rev. Lett. 77 2626-2629

[16] Airapetian A et al. (HERMES) 2000 Phys. Rev. Lett. 84 4047-4051 (Preprint hep-ex/9910062)

[17] Avakian H et al. (CLAS) 2004 Phys. Rev. D69 112004 (Preprint hep-ex/0301005)

[18] Airapetian A et al. (HERMES) 2005 Phys. Rev. Lett. 94012002 (Preprint hep-ex/0408013)

[19] Alexakhin V Y et al. (COMPASS) 2005 Phys. Rev. Lett. 94202002 (Preprint hep-ex/0503002)

[20] Mueller D, Robaschik D, Geyer B, Dittes F M and Horejsi J 1994 Fortschr. Phys. 42101 (Preprint hep-ph/9812448)

[21] Ji X D 1997 Phys. Rev. Lett. 78 610-613 (Preprint hep-ph/9603249)

[22] Radyushkin A V 1996 Phys. Lett. B380 417-425 (Preprint hep-ph/9604317)

[23] Collins J C, Frankfurt L and Strikman M 1997 (Preprint hep-ph/9709336)

[24] Burkardt M 2003 Int. J. Mod. Phys. A18 173-208 (Preprint hep-ph/0207047)

[25] Kotzinian A 1995 Nucl. Phys. B441 234-248 (Preprint hep-ph/9412283)

[26] Mulders P J and Tangerman R D 1996 Nucl. Phys. B461 197-237 (Preprint hep-ph/9510301)

[27] Boer D and Mulders P J 1998 Phys. Rev. D57 5780-5786 (Preprint hep-ph/9711485)

[28] Collins J C 2003 Acta Phys.Polon. B34 3103 (Preprint hep-ph/0304122)

[29] Pasquini B, Cazzaniga S and Boffi S 2008 Phys. Rev. D78 034025 (Preprint hep-ph/0806.2298)

[30] Bacchetta A et al. 2007 JHEP 02093 (Preprint hep-ph/0611265)

[31] Anselmino M, Barone V and Kotzinian A 2011 Phys. Lett. B699 108-118 (Preprint 1102.4214)

[32] Collins J C and Soper D E 1981 Nucl. Phys. B193 381

[33] Ji X, Ma J and Yuan F 2005 Phys. Rev. D71 034005 (Preprint hep-ph/0404183)

[34] Collins J 2011 Foundations of perturbative QCD (Cambridge University Press)

[35] Aybat S M and Rogers T C 2011 Phys.Rev. D83 114042 (Preprint 1101.5057)

[36] Boer D, Gamberg L, Musch B and Prokudin A 2011 JHEP 1110021 (Preprint 1107.5294)

[37] Aybat S M, Collins J C, Qiu J W and Rogers T C 2012 Phys.Rev. D85 034043 (Preprint 1110.6428)

[38] Kang Z B and Prokudin A 2012 Phys.Rev. D85 07400811 pages, 7 figures (Preprint 1201.5427)

[39] Echevarri M G, Idilbi A, Schfer A and Scimemi I 2013 Eur.Phys.J. C73 2636 (Preprint 1208.1281)

[40] Collins J 2014 Int.J.Mod.Phys.Conf.Ser. 251460001 (Preprint 1307.2920)

[41] Sun P and Yuan F 2013 Phys.Rev. D88 034016 (Preprint 1304.5037)

[42] Sun P and Yuan F 2013 Phys.Rev. D88 114012 (Preprint 1308.5003)

[43] Echevarria M G, Idilbi A, Kang Z B and Vitev I 2014 Phys.Rev. D89 074013 (Preprint 1401.5078)

[44] Sun P, Isaacson J, Yuan C P and Yuan F 2014 (Preprint 1406.3073)

[45] INT Workshop INT-14-55W "Studies of 3D Structure of Nucleon," Organizers: Elke Aschenauer, Barbara Pasquini, Harut Avakian, Peter Schweitzer; workshop website: http://www.int.washington.edu/PROGRAMS/14-55w/ 
[46] Bacchetta A, Boglione M, Henneman A and Mulders P J 2000 Phys. Rev. Lett. 85 712-715 (Preprint hep-ph/9912490)

[47] Pobylitsa P V 2003 hep-ph/0301236 (Preprint hep-ph/0301236)

[48] Efremov A, Goeke K and Pobylitsa P 2000 Phys.Lett. B488 182-186 (Preprint hep-ph/0004196)

[49] Hagler P, Musch B U, Negele J W and Schafer A 2009 Europhys. Lett. 8861001 (Preprint hep-lat/0908.1283)

[50] Musch B U, Hagler P, Negele J W and Schafer A 2011 Phys.Rev. D83 094507 (Preprint 1011.1213)

[51] Musch B, Hagler P, Engelhardt M, Negele J and Schafer A 2012 Phys.Rev. D85 094510 (Preprint $1111.4249)$

[52] Ji X 2013 Phys.Rev.Lett. 110262002 (Preprint 1305.1539)

[53] Ji X, Sun P, Xiong X and Yuan F 2014 (Preprint 1405.7640)

[54] Ma Y Q and Qiu J W 2014 (Preprint 1404.6860)

[55] Lin H W, Chen J W, Cohen S D and Ji X 2014 (Preprint 1402.1462)

[56] Jakob R, Mulders P J and Rodrigues J 1997 Nucl. Phys. A626 937-965 (Preprint hep-ph/9704335)

[57] Goldstein G R and Gamberg L 2002 452-454 (Preprint hep-ph/0209085)

[58] Gamberg L P, Goldstein G R and Oganessyan K A 2003 Phys. Rev. D67 071504 (Preprint hep-ph/0301018)

[59] Gamberg L P, Goldstein G R and Schlegel M 2008 Phys. Rev. D77 094016 (Preprint hep-ph/0708.0324)

[60] Bacchetta A, Conti F and Radici M 2008 Phys.Rev. D78 074010 (Preprint 0807.0323)

[61] Lu Z and Schmidt I 2010 Phys.Rev. D82 094005 (Preprint 1008.2684)

[62] She J, Zhu J and Ma B Q 2009 Phys. Rev. D79 054008 (Preprint 0902.3718)

[63] Zhu J and Ma B Q 2011 Phys. Lett. B696 246-251 (Preprint 1104.4564)

[64] Ma B Q 1991 J.Phys. G17 L53-L58 (Preprint 0711.2335)

[65] Wakamatsu M 2009 Phys. Rev. D79 094028 (Preprint 0903.1886)

[66] Schweitzer P, Strikman M and Weiss C 2013 JHEP 1301163 (Preprint 1210.1267)

[67] Lorce C, Pasquini B and Vanderhaeghen M 2011 JHEP 1105041 (Preprint 1102.4704)

[68] Avakian H, Efremov A V, Schweitzer P and Yuan F 2010 Phys. Rev. D81 074035 (Preprint hep-ph/1001.5467)

[69] Avakian H, Efremov A, Schweitzer P and Yuan F 2008 Phys.Rev. D78 114024 (Preprint $0805.3355)$

[70] Efremov A V et al. 2009 Phys. Rev. D80 014021 (Preprint hep-ph/0903.3490)

[71] Efremov A, Schweitzer P, Teryaev O and Zavada P 2010 PoS DIS2010 253 (Preprint 1008.3827)

[72] Efremov A, Schweitzer P, Teryaev O and Zavada P 2011 Phys.Rev. D83 054025 (Preprint 1012.5296)

[73] Kundu R and Metz A 2002 Phys. Rev. D65 014009 (Preprint hep-ph/0107073)

[74] Goeke K, Meissner S, Metz A and Schlegel M 2006 Phys.Lett. B637 241-244 (Preprint hep-ph/0601133)

[75] Meissner S, Metz A and Goeke K 2007 Phys. Rev. D76 034002 (Preprint hep-ph/0703176)

[76] Mukherjee A 2010 Phys. Lett. B687 180-183 (Preprint 0912.1446)

[77] Mukherjee A 2011 Mod.Phys.Lett. A26 2653-2662 (Preprint 1005.2830)

[78] Cloet I C, Bentz W and Thomas A W 2008 Phys. Lett. B659 214-220 (Preprint 0708.3246)

[79] Matevosyan H H, Bentz W, Cloet I C and Thomas A W 2012 Phys.Rev. D85 014021 (Preprint $1111.1740)$

[80] Makke N 4th International Workshop on Transverse Polarisation Phenomena in Hard Processes (Transversity 2014)

[81] Brodsky S J, Hwang D S, Ma B Q and Schmidt I 2001 Nucl.Phys. B593 311-335 (Preprint hep-th/0003082)

[82] Ji X d, Ma J P and Yuan F 2003 Nucl. Phys. B652 383-404 (Preprint hep-ph/0210430) 
[83] Pasquini B, Boffi S and Schweitzer P 2009 Mod.Phys.Lett. A24 2903-2912 (Preprint 0910.1677)

[84] Boffi S, et al. 2009 Phys. Rev. D79 094012 (Preprint hep-ph/0903.1271)

[85] Pasquini B and Schweitzer P 2011 Phys.Rev. D83 114044 (Preprint 1103.5977)

[86] Pasquini B and Schweitzer P 2014 Phys.Rev. D90 014050 (Preprint 1406.2056)

[87] She J and Ma B Q 2011 Phys.Rev. D83 037502 (Preprint 1101.5196)

[88] Lorce C and Pasquini B 2011 Phys.Rev. D84 034039 (Preprint 1104.5651)

[89] Schweitzer P, Teckentrup T and Metz A 2010 Phys. Rev. D81 094019 (Preprint 1003.2190)

[90] Bourrely C, Soffer J and Buccella F 2006 Mod.Phys.Lett. A21 143-150 (Preprint hep-ph/0507328)

[91] Bourrely C, Buccella F and Soffer J 2011 Phys.Rev. D83 074008 (Preprint 1008.5322)

[92] D'Alesio U, Leader E and Murgia F 2010 Phys.Rev. D81 036010 (Preprint 0909.5650)

[93] Teckentrup T, Metz A and Schweitzer P 2009 Mod. Phys. Lett. A24 2950-2959 (Preprint 0910.2567)

[94] Brodsky S J, Hwang D S and Schmidt I 2002 Phys. Lett. B530 99-107 (Preprint hep-ph/0201296)

[95] Bacchetta A, Schafer A and Yang J J 2004 Phys. Lett. B578 109-118 (Preprint hep-ph/0309246)

[96] Lu Z and Ma B Q 2004 Nucl. Phys. A741 200-214 (Preprint hep-ph/0406171)

[97] Lu Z and Schmidt I 2007 Phys.Rev. D75 073008 (Preprint hep-ph/0611158)

[98] Ellis J R, Hwang D S and Kotzinian A 2009 Phys. Rev. D80 074033 (Preprint 0808.1567)

[99] Yuan F 2003 Phys. Lett. B575 45-54 (Preprint hep-ph/0308157)

[100] Courtoy A, Scopetta S and Vento V 2009 Phys.Rev. D79 074001 (Preprint 0811.1191)

[101] Courtoy A, Scopetta S and Vento V 2009 Phys. Rev. D80 074032 (Preprint 0909.1404)

[102] Courtoy A, Fratini F, Scopetta S and Vento V 2008 Phys. Rev. D78 034002 (Preprint 0801.4347 )

[103] Pasquini B and Yuan F 2010 Phys. Rev. D81 114013 (Preprint 1001.5398)

[104] Gamberg L and Schlegel M 2009 Mod. Phys. Lett. A24 2960-2972 (Preprint 0912.5399)

[105] Gamberg L and Schlegel M 2010 Phys. Lett. B685 95-103 (Preprint 0911.1964)

[106] Brodsky S J, Pasquini B, Xiao B W and Yuan F 2010 Phys.Lett. B687 327-330 (Preprint 1001.1163)

[107] Artru X, Czyzewski J and Yabuki H 1997 Z.Phys. C73 527-534 (Preprint hep-ph/9508239)

[108] Czyzewski J 1996 Acta Phys.Polon. 27 1759-1766 (Preprint hep-ph/9606390)

[109] Artru X and Belghobsi Z 2011 AIP Conf.Proc. 1444 97-105

[110] Bacchetta A, Kundu R, Metz A and Mulders P 2001 Phys.Lett. B506 155-160 (Preprint hep-ph/0102278)

[111] Bacchetta A, Kundu R, Metz A and Mulders P J 2002 Phys. Rev. D65 094021 (Preprint hep-ph/0201091)

[112] Gamberg L P, Goldstein G R and Oganessyan K A 2003 Phys.Rev. D68 051501 (Preprint hep-ph/0307139)

[113] Amrath D, Bacchetta A and Metz A 2005 Phys. Rev. D 71114018 (Preprint hep-ph/0504124)

[114] Bacchetta A, Gamberg L P, Goldstein G R and Mukherjee A 2008 Phys. Lett. B659 234-243 (Preprint hep-ph/0707.3372)

[115] Bacchetta A, Metz A and Yang J J 2003 Phys.Lett. B574 225-231 (Preprint hep-ph/0307282)

[116] Matevosyan H H, Thomas A W and Bentz W 2011 Phys.Rev. D83 114010 (Preprint 1103.3085)

[117] Matevosyan H H, Thomas A W and Bentz W 2012 Phys.Rev. D86 034025 (Preprint 1205.5813)

[118] Matevosyan H H, Kotzinian A and Thomas A W 2014 Phys.Lett. B731 208-216 (Preprint 1312.4556)

[119] Radici M 2012 Nuovo Cim. C035N2 69-77 (Preprint 1111.3383)

[120] Anselmino M et al. 2005 Phys. Rev. D71 074006 (Preprint hep-ph/0501196)

[121] Collins J C et al. 2006 Phys. Rev. D73 014021 (Preprint hep-ph/0509076)

[122] Collins J C, Soper D E and Sterman G F 1985 Nucl.Phys. B250 199

[123] Davies C, Webber B and Stirling W J 1985 Nucl.Phys. B256 413

[124] Ladinsky G and Yuan C 1994 Phys.Rev. D50 4239 (Preprint hep-ph/9311341)

[125] Landry F, Brock R, Ladinsky G and Yuan C 2001 Phys.Rev. D63 013004 (Preprint 
hep-ph/9905391)

[126] Landry F, Brock R, Nadolsky P M and Yuan C 2003 Phys.Rev. D67 073016 (Preprint hep-ph/0212159)

[127] Konychev A V and Nadolsky P M 2006 Phys.Lett. B633 710-714 (Preprint hep-ph/0506225)

[128] Bozzi G, Catani S, de Florian D and Grazzini M 2006 Nucl.Phys. B737 73-120 (Preprint hep-ph/0508068)

[129] Dulat S, Hou T J, Gao J, Huston J, Nadolsky P et al. 2014 Phys.Rev. D89 113002 (Preprint 1310.7601)

[130] Hautmann F 2013 Acta Phys.Polon. B44 761-778

[131] Mantry S and Petriello F 2011 Phys.Rev. D84 014030 (Preprint 1011.0757)

[132] Guzzi M, Nadolsky P M and Wang B 2014 Phys.Rev. D90 014030 (Preprint 1309.1393)

[133] Efremov A V, Goeke K, Menzel S, Metz A and Schweitzer P 2005 Phys. Lett. B612 233-244 (Preprint hep-ph/0412353)

[134] Arnold S, Efremov A V, Goeke K, Schlegel M and Schweitzer P 2008 hep-ph/0805.2137 (Preprint hep-ph/0805.2137)

[135] Anselmino M, Boglione M, D'Alesio U, Kotzinian A, Melis S et al. 2009 Eur.Phys.J. A39 89-100 (Preprint 0805.2677)

[136] Vogelsang W and Yuan F 2005 Phys. Rev. D72 054028 (Preprint hep-ph/0507266)

[137] Efremov A V, Goeke K and Schweitzer P 2006 Phys. Rev. D73 094025 (Preprint hep-ph/0603054)

[138] Anselmino M, Boglione M, D’Alesio U, Kotzinian A, Murgia F et al. 2007 Phys.Rev. D75 054032 (Preprint hep-ph/0701006)

[139] Anselmino M, Boglione M, D'Alesio U, Melis S, Murgia F et al. 2013 Phys.Rev. D87 094019 (Preprint 1303.3822)

[140] Boer D 1999 Phys.Rev. D60 014012 (Preprint hep-ph/9902255)

[141] Lu Z, Ma B Q and Schmidt I 2006 Phys.Lett. B639 494-498 (Preprint hep-ph/0702006)

[142] Zhang B, Lu Z, Ma B Q and Schmidt I 2008 Phys. Rev. D77 054011 (Preprint hep-ph/0803.1692)

[143] Lu Z and Schmidt I 2010 Phys. Rev. D81 034023 (Preprint 0912.2031)

[144] Barone V, Melis S and Prokudin A 2010 Phys. Rev. D81 114026 (Preprint 0912.5194)

[145] Anselmino M, Boglione M, Collins J, D'Alesio U, Efremov A et al. 2005 236-243 (Preprint hep-ph/0511017)

[146] Collins J C et al. 2006 Phys. Rev. D73 094023 (Preprint hep-ph/0511272)

[147] Bianconi A and Radici M 2005 Phys.Rev. D71 074014 (Preprint hep-ph/0412368)

[148] Sissakian A, Shevchenko O, Nagaytsev A and Ivanov O 2009 Eur.Phys.J. C59 659-673 (Preprint $0807.2480)$

[149] Anselmino M, Boglione M, D'Alesio U, Melis S, Murgia F et al. 2009 Phys.Rev. D79 054010 (Preprint 0901.3078)

[150] Barone V, Melis S and Prokudin A 2010 Phys.Rev. D82 114025 (Preprint 1009.3423)

[151] Lu Z and Schmidt I 2011 Phys.Rev. D84 094002 (Preprint 1107.4693)

[152] Aybat S M, Prokudin A and Rogers T C 2012 Phys.Rev.Lett. 108242003 (Preprint 1112.4423)

[153] Collins J C and Rogers T C 2013 Phys.Rev. D87 034018 (Preprint 1210.2100)

[154] Kang Z B, Vitev I and Xing H 2013 Phys.Rev. D87 034024 (Preprint 1212.1221)

[155] Kang Z B and Xiao B W 2013 Phys.Rev. D87 034038 (Preprint 1212.4809)

[156] Anselmino M, Boglione M and Melis S 2012 Phys.Rev. D86 014028 (Preprint 1204.1239)

[157] Bacchetta A and Prokudin A 2013 Nucl.Phys. B875 536-551 (Preprint 1303.2129)

[158] Boer D 2013 Nucl.Phys. B874 217-229 (Preprint 1304.5387)

[159] Adolph C et al. (COMPASS) 2013 Eur.Phys.J. C73 2531 (Preprint 1305.7317)

[160] Signori A, Bacchetta A, Radici M and Schnell G 2013 JHEP 1311194 (Preprint 1309.3507)

[161] Anselmino M, Boglione M, Gonzalez H J, Melis S and Prokudin A 2014 JHEP 1404005 (Preprint $1312.6261)$

[162] Hautmann F, Jung H, Krmer M, Mulders P, Nocera E et al. 2014 (Preprint 1408.3015) 
[163] Aghasyan M and Avakian H 2014 Phys.Part.Nucl. 45 126-128 (Preprint 1302.1080)

[164] Asaturyan R, Ent R, Mkrtchyan H, Navasardyan T, Tadevosyan V et al. 2012 Phys.Rev. C85 015202 (Preprint 1103.1649)

[165] Aghasyan M, Avakian H, De Sanctis E, Gamberg L, Mirazita M et al. 2014 (Preprint 1409. 0487)

[166] Akushevich I, Shumeiko N and Soroko A 1999 Eur. Phys. J. C10 681-687 (Preprint hep-ph/9903325)

[167] Akushevich I, Ilyichev A and Osipenko M 2009 Phys.Lett. B672 35-44 (Preprint 0711.4789)

[168] Ingelman G, Edin A and Rathsman J 1997 Comput.Phys.Commun. 101 108-134 (Preprint hep-ph/9605286)

[169] Kotzinian A 2005 Proceedings of Transversity 2005, 228-235 (Preprint hep-ph/0510359)

[170] Kotzinian A 2005 (Preprint hep-ph/0504081)

[171] https://wiki.bnl.gov/eic/index.php/LEPTO-PHI URL https://wiki.bnl.gov/eic/index.php/LEPTO-PHI

[172] Kotzinian A, Matevosyan H H and Thomas A W 2014 Phys.Rev.Lett. 113062003 (Preprint 1403.5562)

[173] Kotzinian A, Matevosyan H H and Thomas A W 2014 Phys. Rev. D 90(7) 074006 (Preprint 1405.5059)

[174] Artru X and Collins J C 1996 Z. Phys. C69 277-286 (Preprint hep-ph/9504220)

[175] Artru X 2002 arXiv:hep-ph/0207309 (Preprint hep-ph/0207309)

[176] Artru X 2010 (Preprint 1001.1061)

[177] Bacchetta A and Radici M 2006 Phys. Rev. D74 114007 (Preprint hep-ph/0608037)

[178] Matevosyan H H, Thomas A W and Bentz W 2014 EPJ Web Conf. 6606014 (Preprint $1307.8125)$

[179] Matevosyan H H, Thomas A W and Bentz W 2013 Phys.Rev. D88 094022 (Preprint 1310.1917)

[180] https://herwig.hepforge.org/trac/ URL https://herwig.hepforge.org/trac/

[181] Aidala C, Field B, Gamberg L and Rogers T 2014 Phys.Rev. D89 094002 (Preprint 1401.2654)

[182] Mulders P J and Rodrigues J 2001 Phys. Rev. D63 094021 (Preprint hep-ph/0009343)

[183] Anselmino M, Boglione M, D'Alesio U, Leader E and Murgia F 2004 Phys.Rev. D70 074025 (Preprint hep-ph/0407100)

[184] Ji X d, Ma J P and Yuan F 2005 JHEP 0507020 (Preprint hep-ph/0503015)

[185] Anselmino M, D'Alesio U, Melis S and Murgia F 2006 Phys.Rev. D74 094011 (Preprint hep-ph/0608211)

[186] Boer D, Brodsky S J, Mulders P J and Pisano C 2011 Phys.Rev.Lett. 106132001 (Preprint 1011.4225)

[187] Metz A and Zhou J 2011 Phys.Rev. D84 051503 (Preprint 1105.1991)

[188] Sun P, Xiao B W and Yuan F 2011 Phys.Rev. D84 094005 (Preprint 1109.1354)

[189] Boer D, den Dunnen W J, Pisano C, Schlegel M and Vogelsang W 2012 Phys.Rev.Lett. 108 032002 (Preprint 1109.1444)

[190] Dominguez F, Qiu J W, Xiao B W and Yuan F 2012 Phys.Rev. D85 045003 (Preprint 1109.6293)

[191] Pisano C, Boer D, Brodsky S J, Buffing M G and Mulders P J 2013 JHEP 1310024 (Preprint 1307.3417)

[192] den Dunnen W J, Lansberg J P, Pisano C and Schlegel M 2014 Phys.Rev.Lett. 112212001 (Preprint 1401.7611)

[193] Boer D and den Dunnen W J 2014 Nucl.Phys. B886 421-435 (Preprint 1404.6753)

[194] Burkardt M, Miller C and Nowak W 2010 Rept.Prog.Phys. 73016201 (Preprint 0812.2208)

[195] Aidala C A, Bass S D, Hasch D and Mallot G K 2013 Rev.Mod.Phys. 85 655-691 (Preprint 1209.2803)

[196] Peng J C and Qiu J W 2014 Prog.Part.Nucl.Phys. 76 43-75 (Preprint 1401.0934) 